\title{
FET Noise-Parameter Determination Using a Novel Technique Based on 50- $\Omega$ Noise-Figure Measurements
}

\author{
Antonio Lázaro, Lluís Pradell, Member, IEEE, and Juan M. O’Callaghan
}

\begin{abstract}
A novel method for measuring the four noise parameters of a field-effect transistor (FET) is presented. It is based on the determination of its intrinsic noise matrix elements $\left[C_{11}^{\mathrm{INT}}\right.$, $\left.C_{22}^{\mathrm{INT}}, \operatorname{Re}\left(C_{12}^{\mathrm{INT}}\right), \operatorname{Im}\left(C_{12}^{\mathrm{INT}}\right)\right]$ by fitting the measured device noise figure for a matched source reflection coefficient $\left(F_{50}\right)$ at a number of frequency points, thus, a tuner is not required. In contrast to previous works, no restrictive assumptions are made on the intrinsic noise sources. The receiver full-noise calibration is easily performed by using a set of coaxial and on-wafer standards that are commonly available in a microwave laboratory, thus, an expensive broad-band tuner is not required for calibration either. On-wafer experimental verification up to $26 \mathrm{GHz}$ is presented and a comparison with other $F_{50}$-based and tunerbased methods is given. As an application, the dependence of the FET intrinsic noise sources as a function of the bias drain-current and gate-length is obtained.
\end{abstract}

Index Terms - Noise measurements, noise modeling, noise parameters.

\section{INTRODUCTION}

$\mathbf{T}$ HE full-noise characterization of a field-effect transistor (FET) requires the determination of its four noise parameters: minimum noise figure $F_{\min }$, noise resistance $R_{n}$, and optimum source reflection coefficient $\Gamma_{\text {opt }}$ (magnitude and phase). The measurement of the noise parameters in the microwave frequency range is usually performed by measuring the device noise figure for a minimum of four source reflection coefficients produced with a tuner [1]-[6]. Although this method gives accurate results, it has the following drawbacks.

1) Broad-band tuners are generally very expensive and the measurements are time consuming.

2) Some tuners may have little flexibility in selecting the source coefficient patterns [3]-[-8] required.

3) It is very sensitive to experimental errors whenever $\left|\Gamma_{\text {opt }}\right|$ is high $(>0.7)$.

This situation is commonly found in microwave FET's at low frequencies (which can be up to $10 \mathrm{GHz}$ ) or for low drain-bias currents.

Other successful techniques make use of a transistor equivalent circuit to provide additional information to reduce complexity in the measurement procedure. In these techniques, the

Manuscript received May 19, 1998; revised October 30, 1998. This work was supported by the Spanish CICYT under research project TIC93-672-C0403 and under research project TIC97-1129-C04-04.

The authors are with the Department of Signal Theory and Communications, Polytechnic University of Catalunya, 08034 Barcelona, Spain.

Publisher Item Identifier S 0018-9480(99)01952-3.

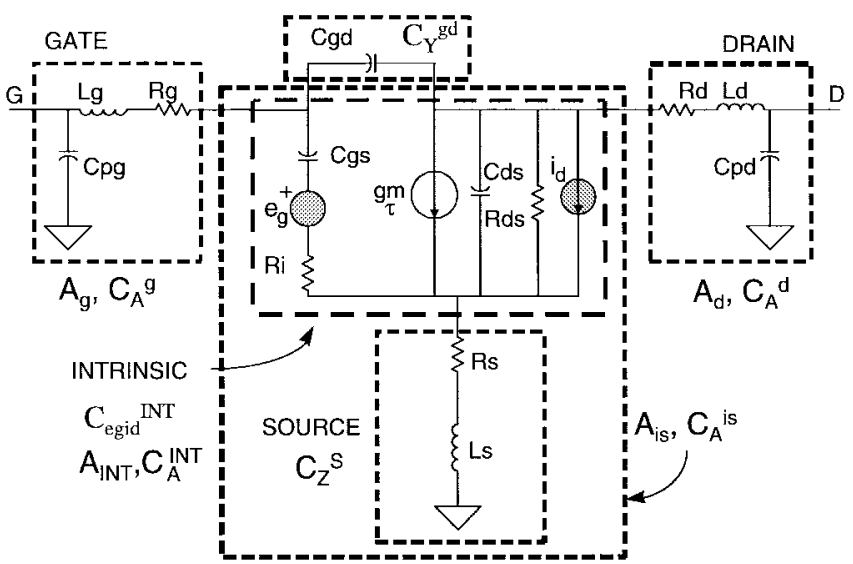

Fig. 1. FET equivalent circuit, including the voltage-current noise-source configuration.

noise parameters are characterized by determining the intrinsic noise correlation matrix $\left[C^{\mathrm{INT}}\right]:\left[C_{11}^{\mathrm{INT}}, C_{22}^{\mathrm{INT}}, \operatorname{Re}\left(C_{12}^{\mathrm{INT}}\right)\right.$, $\left.\operatorname{Im}\left(C_{12}^{\mathrm{INT}}\right)\right][8]$ through a number of simplifying assumptions. If the intrinsic noise sources are arranged in a voltage-current (hybrid) configuration $\left(e_{g}, i_{d}\right)$, as shown in Fig. $1,\left[C^{\mathrm{INT}}\right]$ is basically frequency-independent [10]-[16]. Therefore, the intrinsic noise matrix can be obtained from a measurement of the four noise parameters at a single frequency in a deembedding procedure [14]. A further simplification assumes no correlation between sources (Pospieszalski's model [10]). In that case, only two frequency-independent noise constants (gate temperature $T_{g}$ and drain temperature $T_{d}$ ) have to be determined. Moreover, other authors [12], [15] assume that $T_{g}$ equals the physical temperature, in which case only one parameter $\left(T_{d}\right)$ must be determined to extract the four noise parameters. This can be done from the measurement of the transistor noise figure, with a matched $(50 \Omega)$ source reflection coefficient $\left(F_{50}\right)$, as proposed by Tasker et al. [12], thus, a tuner is not required. Alternatively, Dambrine et al. [9] use the current-current intrinsic noise-source configuration (also called admittance or $R, P, C$ model) with a number of simplifying assumptions (in particular, the correlation coefficient $C$ is assumed to be purely imaginary and related to parameters $R, P$ by the approximate expression $C \cong(R / P)^{1 / 2}$ ), to determine the four noise parameters from the measurement of $F_{50}$ as a function of frequency. While the noise-parameters determination based on $F_{50}$ is of interest due to its simplicity, 
improvements in accuracy are expected if no simplifying assumptions on the intrinsic noise sources are made.

The purpose of this paper is to propose a new method to determine the intrinsic (voltage-current or hybrid) FET noise matrix, based on the measurement of $F_{\tilde{5} 0}$ for a redundant number of frequency points $N$. In contrast with previous works [9], [12], no restrictions are imposed on the intrinsic noise sources, in particular, the correlation coefficient is not supposed to be zero. This assumption is supported by recent works [14], [16], [17]. Moreover, a smooth low-order polynomial frequency dependence is allowed on the intrinsic noise matrix elements.

\section{NOISE-PARAMETER-EXTRACTION PROCEDURE From $F_{50}$}

The procedure is based on the FET equivalent circuit shown in Fig. 1, which includes its intrinsic noise sources $e_{g}, i_{d}$ (hybrid configuration). The FET $S$-parameters and the parasitic elements are known from measurements, and the transistor $F_{50}$ is measured at $N$ frequency points in a broad range (2-26 GHz), as explained in Section IV. The noise-parameter characterization method proposed in this paper consists of determining the intrinsic noise correlation matrix $C_{\text {egid }}^{\mathrm{INT}}$ from $F_{50}$. The basics of this procedure are detailed below.

The transistor noise figure for the measured source impedance $Z_{s}^{i}=R_{s}^{i}+j X_{s}^{i}$ (corresponding to the $i$ th frequency) can be expressed as a function of its cascade noise matrix $C_{A}$ [8] as follows:

$$
F\left(Z_{s}^{i}\right)=1+\frac{1}{4 k T_{0} \Delta f \operatorname{Re}\left(Z_{s}^{i}\right)} \cdot\left[1 Z_{s}^{i^{*}}\right] \cdot C_{A} \cdot\left[\begin{array}{c}
1 \\
Z_{s}^{i}
\end{array}\right]
$$

where $k$ is the Boltzmann constant and $T_{o}=290 \mathrm{~K}$.

According to the equivalent circuit in Fig. 1, $C_{A}$ can be split in its intrinsic and extrinsic (thermal noise due to the parasitic elements) contributions [18] $C_{A}=C_{A}^{\mathrm{EXT}}+C_{A}^{\mathrm{INT}}$ where

$$
\begin{aligned}
C_{A}^{\mathrm{EXT}}= & C_{A}^{g}+\left(A_{g} A_{i s}\right) C_{A}^{d}\left(A_{g} A_{i s}\right)^{+}+\left(A_{g} P_{Z A}\right) C_{Z}^{s}\left(A_{g} P_{Z A}\right)^{+} \\
& +\left(A_{g} P_{Z A} P_{Y Z}\right) C_{Y}^{\mathrm{gd}}\left(A_{g} P_{Z A} P_{Y Z}\right)^{+} \\
C_{A}^{\mathrm{INT}}= & P \cdot C_{\text {egid }}^{\mathrm{INT}} \cdot P^{+} \\
P= & A_{g} P_{Z A} P_{Y Z} P_{H Y}, P_{H Y}=\left[\begin{array}{ll}
-Y_{11}^{\mathrm{INT}} & 0 \\
-Y_{21}^{\mathrm{INT}} & 1
\end{array}\right]
\end{aligned}
$$

The meaning of the matrices in (2)-(4) are (see Fig. 1) as follows:

$A_{g} \quad A B C D$ (cascade) matrix of the gate two-port;

$A_{\text {is }} A B C D$ matrix of the series connection of the source two-port and the intrinsic FET;

$P_{n m} \quad$ conversion matrix from $n$ to $m$ noise-source configuration (for details see [8, Table I]);

$C_{Z}^{S}$ impedance noise matrix of the source passive twoport;

$C_{y}^{\text {gd }}$ admittance noise matrix of the gate-to-drain passive two-port;

$C_{A}^{g} A B C D$ (cascade) noise matrix of the gate passive two-port;

$C_{A}^{d} A B C D$ (cascade) noise matrix of the drain passive two-port;

$C_{\text {egid }}^{\mathrm{INT}}$ intrinsic voltage-current (hybrid) noise matrix.
Note that $C_{A}^{\mathrm{EXT}}$ and $P_{n m}$ are functions of the $S$-parameters and the room temperature only.

A linear frequency dependence for the elements of $C_{\text {egid }}^{\mathrm{INT}}$ is assumed, although higher order polynomials $\left(C_{i j}^{\mathrm{INT}}=\right.$ $\left.C_{i j}^{0}+C_{i j}^{1} f+\cdots\right)$ have also been considered with no significant improvement of results. Other types of noise $(1 / f, g-r)$ are present, mainly at low frequencies (up to $1 \mathrm{GHz}$, depending on the transistor) [27], and can be included in $C_{\text {egid }}^{\mathrm{INT}}$ by adding terms of the form $C_{i j}^{\alpha} / f^{\alpha}$ and $C_{i j}^{\beta} /\left(1+\left(f^{2} / f_{c}^{2}\right)\right)$, where $f_{c}$ is the cutoff frequency of the trap process and $C_{i j}^{\alpha}, C_{i j}^{\beta}$ are coefficients which have to be estimated from low-frequency measurements as a last step in the optimization procedure explained below. Since the lowest measurement frequency of the experimental setup is $2 \mathrm{GHz}$ (see Section III), this dependence has not been observed in the transistors measured and will not be considered in the derivation. Shot noise is also a low-frequency effect that can also be included by adding noise current sources to the admittance intrinsic noise matrix, as explained below. Substituting (2)-(4) into (1), the following overdetermined linear equation system is obtained for $C_{\text {egid }}^{\mathrm{INT}}$ :

$$
\left[y_{i}\right]=[M] \cdot[X], \quad i=1, \cdots, N
$$

where $N$ is the number of measured frequency points. The left-hand side of (5) depends on the measured noise figure $F\left(Z_{S}^{i}\right)$ at every frequency point $f_{i}\left(F_{50}\right)$, and the extrinsic noise contribution:

$y_{i}=4 k T_{0} \Delta f \cdot\left(F\left(Z_{S}^{i}\right)-1\right) \cdot \operatorname{Re}\left(Z_{S}^{i}\right)-\left[1 Z_{S}^{i^{*}}\right] \cdot C_{A}^{\mathrm{EXT}} \cdot\left[1 Z_{S}^{i}\right]^{T}$.

The right-hand side of (5), $[X]$, is the unknown intrinsic correlation matrix $C_{\text {egid }}^{\mathrm{INT}}$ arranged as a column vector and expanded as a frequency polynomial $[X]=\left[C^{0}\right]+\left[f_{i}\right]\left[C^{1}\right]+$ $\cdots+\left[f_{i}^{k}\right]\left[C^{k}\right]+\cdots$ whose coefficients are given by $\left[C^{k}\right]=$ $\left[C_{11}^{k}, C_{22}^{k}, \operatorname{Re} C_{12}^{k}, \operatorname{Im} C_{12}^{k}\right]^{T}$.

The elements of matrix $[M]$ only depend on the FET $[S]$ parameters, and the source impedances. They are given by

$$
\begin{aligned}
M_{i l}= & \left|P_{11}\right|^{2}+\left|Z_{s}^{i}\right|^{2}\left|P_{21}\right|^{2}+2 R_{S}^{i} \operatorname{Re}\left(P_{11} P_{21}^{*}\right) \\
& -2 X_{S}^{i} \operatorname{Im}\left(P_{11} P_{21}^{*}\right) \\
M_{i 2}= & \left|M_{12}\right|^{2}+\left|Z_{S}^{i}\right|^{2}\left|P_{22}\right|^{2}+2 R_{S}^{i} \operatorname{Re}\left(P_{12} P_{22}^{*}\right) \\
& -2 X_{S}^{i} \operatorname{Im}\left(P_{12} P_{22}^{*}\right) \\
M_{i 3}= & 2 \operatorname{Re}\left(P_{11} P_{12}^{*}\right)+2\left|Z_{S}^{i}\right|^{2} \operatorname{Re}\left(P_{12} P_{22}^{*}\right) \\
& +2 R_{S}^{i} \operatorname{Re}\left(P_{12} P_{21}^{*}+P_{11} P_{22}^{*}\right) \\
M_{i 4}= & -2 \operatorname{Im}\left(P_{11} P_{12}^{*}\right)-2\left|Z_{S}^{i}\right|^{2} \operatorname{Im}\left(P_{21} P_{22}^{*}\right) \\
& -2 R_{S}^{i} \operatorname{Re}\left(P_{11} P_{21}^{*}-P_{21} P_{22}^{*}\right) .
\end{aligned}
$$

The linear system (5) is solved for $C_{i j}^{\mathrm{INT}}$ by a fitting procedure that involves the estimation of a large number of parameters: $C_{i j}^{k}(k=0,1, \cdots)$. This multivariable optimization problem is divided into subproblems with a smaller number of variables (one or two). This method has been applied in the literature to the extraction of equivalent circuits to avoid local minima in the error function [19]. The noise matrix elements which produce the highest sensitivity in the noise parameters, i.e., $C_{i j}^{0}$, are first computed by assuming no frequency dependence on $C_{i j}^{\mathrm{INT}}\left(C_{i j}^{k}=0, k=1,2, \cdots\right)$. With 
this assumption, (5) is solved for $C_{i j}^{0}$ by least squares using pseudo-inverse calculation. The values obtained for $C_{i j}^{0}$ are used as initial values in a simplex algorithm that estimates $C_{i j}^{0}$ and $C_{i j}^{1}$ for the best fit of the computed $F_{50}(5)-(10)$ to the measured $F_{50}$ using a robust Huber error function [20].

Note that other noise-source configurations can be used in (5), simply by changing the matrix $P_{\mathrm{HY}}$; e.g., the admittance model (current-current) $C_{\text {igsids }}^{\mathrm{INT}}$ can be used if $P_{\mathrm{HY}}$ is set to the identity matrix. In this case, the frequency dependence of the intrinsic noise matrix can be approximated by $C_{11}^{\mathrm{INT}}=$ $C_{11}^{2} \cdot f^{2}, C_{12}^{\mathrm{INT}}=C_{12}^{1} \cdot f$ and $C_{22}^{\mathrm{INT}}=C_{22}^{0}+C_{22}^{1} \cdot f[9]$, [13]. Shot noise can also be included by adding a current noise source in parallel to capacitance $C_{\mathrm{gs}}$, with a spectral density of $2 q I_{\mathrm{GS}}\left(\mathrm{A}^{2} / \mathrm{Hz}\right)$, and a current noise source in parallel to capacitance $C_{\mathrm{gd}}$, with a spectral density of $2 q I_{\mathrm{GD}}$ $\left(\mathrm{A}^{2} / \mathrm{Hz}\right)$, where $I_{\mathrm{GS}}$ and $I_{\mathrm{GD}}$ are de currents flowing through gate-source and gate-drain diodes, respectively. Since $I_{\mathrm{GS}}$, $I_{\mathrm{GD}}$, and $g_{\mathrm{did}}$ (gate-source diode conductance) are known from dc measurements, the shot noise contribution is treated as extrinsic by adding a new term in (2). This term is written as $\left(P_{\mathrm{SHOT}} \cdot C_{Y}^{\mathrm{SHOT}} \cdot P_{\mathrm{SHOT}}^{+}\right)$, where

$$
\begin{aligned}
& C_{Y}^{\mathrm{SHOT}} \\
& =2 q \Delta f\left[\begin{array}{cc}
\frac{I_{\mathrm{GS}}}{\left(1+g_{\mathrm{did}} R_{i}\right)^{2}+\left(\omega C_{\mathrm{gs}} R_{i}\right)^{2}}+I_{\mathrm{GD}} & -I_{\mathrm{GD}} \\
-I_{\mathrm{GD}} & I_{\mathrm{GD}}
\end{array}\right]
\end{aligned}
$$

and $P_{\mathrm{SHOT}}=A_{g} P_{Z A} P_{Y Z}$. Shot noise effects are only noticeable for frequencies below a cutoff frequency $f_{c}$, which can be estimated as [28]

$$
f_{c} \cong \frac{1}{2 \pi} \sqrt{\frac{2 q I_{\mathrm{GS} g m}}{4 k T_{0} C_{\mathrm{gg}}^{2} R\left(1-C^{2}\right)}}
$$

where $R, C$ are coefficients of the admittance model [9]. For the dc bias and frequency band $(2-26 \mathrm{GHz})$ used in this paper, this effect is negligible.

\section{CAlibration Procedure}

Fig. 2 shows the experimental setup. It is composed by a wafer-probe station (SUMMIT-9000, Cascade-Microtech), an automatic network analyzer (ANA) HP 8510B, up to $40 \mathrm{GHz}$, a noise measurement system (NMS) HP 8970B/8971C, up to $26.5 \mathrm{GHz}$, and an electronic broad-band noise source HP 346C, up to $26.5 \mathrm{GHz}$. The NMS includes a wide-band low-noise amplifier. An input and output switch are used to automatically select the ANA for $S$-parameters and reflection-coefficient measurements or the NMS for noise power measurement without disconnecting the system. Any connection/disconnection would degrade the measurement accuracy.

To fully calibrate the receiver noise contribution at plane 2 , its four noise parameters are needed. Obviously, the procedure explained in Section II cannot be used in this case, but only a conventional, tuner-based method. However, since the receiver front-end is a well-matched low-noise amplifier (the magnitude of its $\Gamma_{\mathrm{opt}}$ is small), there is no need for automatic, expensive tuners. In fact, a "manual" tuner has been used here, composed of coaxial $(3.5-\mathrm{mm})$ standards, commonly found in a microwave laboratory, which are connected to

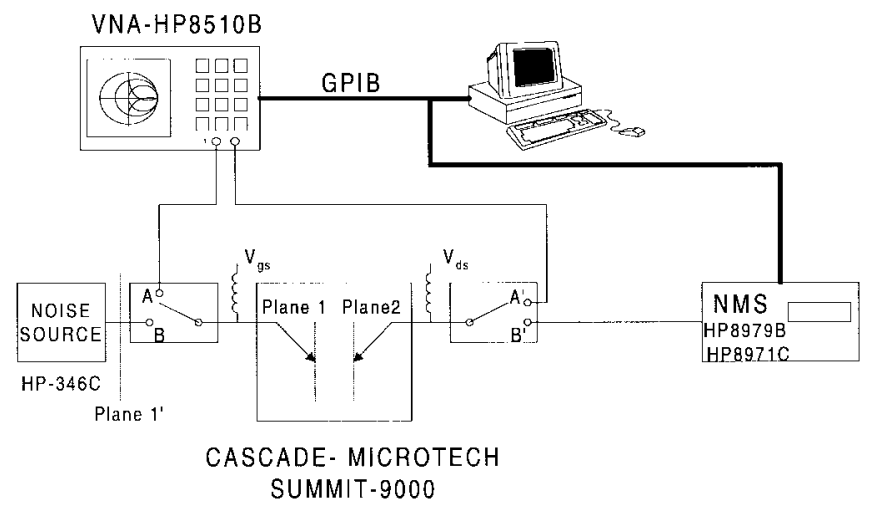

Fig. 2. Measurement setup.

TABLE I

ImPEDANCES Used in the "MANuAL" Receiver CALIBRation

\begin{tabular}{ll}
\hline Standlard at plane B (coaxial 3.5mm) & Standard at planes 1-2 (on wafer) \\
\hline Noise Source Hot/Cold & Thru \\
Short & Thru \\
Match & Thru \\
Short+coaxial attenuator IdB & Thru \\
Short+ coaxial attenuator $6 \mathrm{~dB}$ & Thru \\
Short+ coaxial attenuator $10 \mathrm{~dB}$ & Thru \\
Short+cable & Thru \\
Short & Transmission Line $1 \mathrm{~mm}$ \\
Short & Transmission Line $2 \mathrm{~mm}$ \\
- & Short \\
- & Match \\
- & Coplanar attenuator $10 \mathrm{~dB}$ \\
\hline
\end{tabular}

plane $1^{\prime}$. As more than four source reflection coefficients are needed at the receiver plane 2 to guarantee a good accuracy in the determination of receiver noise parameters, additional onwafer elements (offset line lengths, attenuators, and matched loads) are introduced between the probe tips (planes 1-2). Table I shows the coaxial/on-wafer elements that have been selected to produce a convenient set of (tuner) source points. Provided that their reflection coefficients are accurately measured, the impedances need not be either the same (for different setups) or repeatable (for a given setup). In fact, the delivered noise powers and reflection coefficients are measured successively, as a function of frequency, for every impedance. After the measurement is completed, the impedances will not be connected any more. All tuner measurements are performed at room temperature.

The calibration procedure is as follows. First, with the input and output switches at positions $A$ and $A^{\prime}$ respectively, a twoport, on-wafer line reflect reflect match (LRRM) calibration [24] is performed at the probe tips (on-wafer planes 1-2 in Fig. 2) to calibrate the ANA. Then the input switch is set to position $B$ and a one-port coaxial open short load (OSL) calibration is performed at plane $1^{\prime}$ with a planar "thru" line (1-ps delay) connecting planes 1 and 2. The noise source is connected to plane $1^{\prime}$ and set to its "hot" state. Using the error terms computed in both calibrations, its reflection coefficient and receiver reflection coefficient are measured (switches set 

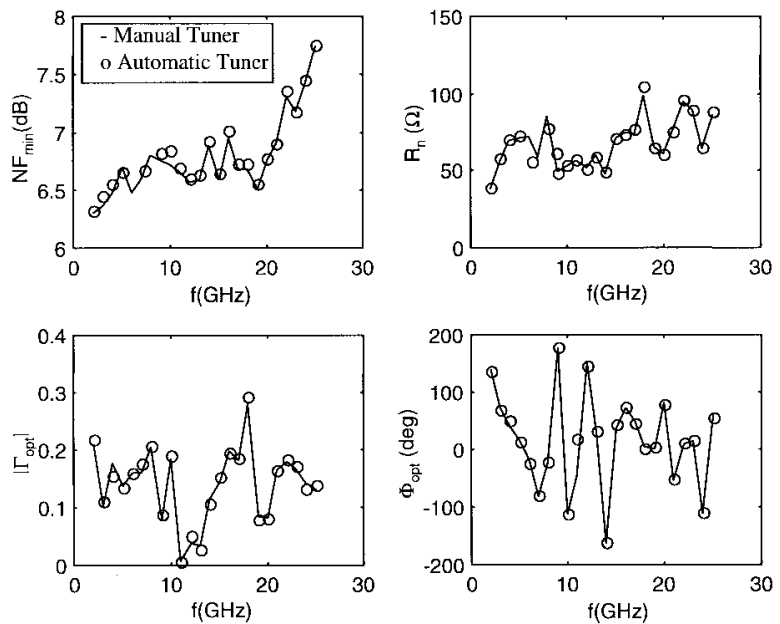

Fig. 3. Measured receiver noise parameters: with manual tuner using the method proposed in [3] (-) and using an automatic tuner (NPTS commercial system $)^{1}(0)$.

to positions $B A^{\prime}$ and $A B^{\prime}$, respectively) and referred to planes 1 and 2, respectively. The switches are then set to positions $B B^{\prime}$ and the noise power delivered by the noise source to the receiver is measured. The noise source is disconnected, the tuner standards are successively connected to plane $1^{\prime}$, and their reflection coefficients and noise powers are measured. With all the collected data, the four receiver noise parameters are determined by following the method proposed in [29]. Alternatively, the method proposed by Davidson [5] has also been used. Results are very similar, as expected whenever the receiver is well matched [29]. Fig. 3 shows the experimental results. The receiver noise parameters measured with the "manual" tuner are plotted and compared to those obtained with an automatic broad-band tuner included in a commercial noise-parameter measurement system (NPTS) from CascadeMicrotech. ${ }^{1}$ Only very small differences can be observed, which are within the measurement uncertainty $[ \pm 0.15-0.2-$ dB root-mean-square (rms)]. Furthermore, the "manual" tuner calibration is very repeatable, as shown in Fig. 4, where the mean value and standard deviation of the receiver noise parameters, measured 16 times in one year, are plot as a function of frequency. This is expected since the measured short-term-gain (3-h) stability of the setup is less than 0.05 $\mathrm{dB}$. The small deviations can be explained by a combination of the measurement uncertainty and fluctuations of the setup characteristics in time.

To summarize, the advantages of a "manual" tuner calibration for the receiver full-noise calibration are availability, adaptability to the particular user needs (measurement range, setup configuration), and measurement accuracy. Moreover, because only $8-12$ source reflection coefficients are required, the calibration time is only slightly greater than using an automatic tuner.

Alternatively, other calibration techniques which do not require a standard tuner have been proposed [25] and could be used here as well.

\footnotetext{
${ }^{1}$ NPTS-26 System, Cascade-Microtech, Inc., Beaverton, OR 97005 USA.
}
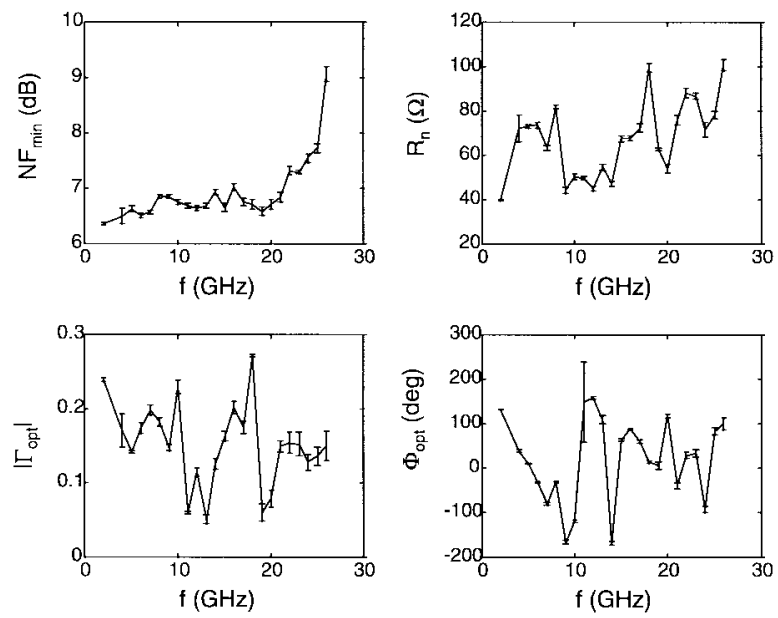

Fig. 4. Measured receiver noise parameters: mean value and standard deviation over 16 measurement sessions in one year.

\section{Transistor Measurement Procedure}

After the receiver calibration, the FET is inserted between the on-wafer planes $1-2$ in Fig. 2. The FET is biased in the active region and its $S$-parameters are measured to $40 \mathrm{GHz}$ for a number of drain-bias currents The small-signal equivalentcircuit extrinsic (bias-independent) elements $\left(R_{g}, R_{s}, R_{d}\right.$, $\left.L_{g}, L_{s}, L_{d}, C_{\mathrm{pg}}, C_{\mathrm{pd}}, C_{\mathrm{gd}}\right)$ are obtained by "cold FET" measurements (see, e.g., [26]). From the FET $S$-matrix and the extrinsic-element values, the intrinsic $Y$-matrix $\left[Y_{i j}^{\mathrm{INT}}\right.$ in (4)] is computed at every bias point. The intrinsic (biasdependent) element values need not be known. The intrinsic $Y$-matrix and the extrinsic-element values are used to compute the $A B C D$, correlation, and conversion matrices required (see Section II). The noise source is then connected to plane $1^{\prime}$ and switched between its hot and cold states. Noise powers $P_{\mathrm{HOT}}^{i}$, $P_{\text {COLD }}^{i}$ delivered by the transistor at the $i$ th frequency $(i=$ $1,2, \cdots, N)$ are measured in the whole noise measurement range $(2-26 \mathrm{GHz})$, for the same bias points. Commercial noise sources have slightly different reflection coefficients in their hot and cold states, but the variation is very small (typically $|\Delta \Gamma|<0.015 \mathrm{~dB})$. Neglecting this effect, the FET noise figure $F_{\text {TRT }}\left(\Gamma_{S}^{i}\right)$ is computed at the $i$ th frequency $(i=1,2, \cdots, N)$ as

$F_{\mathrm{TRT}}\left(\Gamma_{S}^{i}\right)=\frac{P_{\mathrm{COLD}}^{i} \cdot\left(T_{h}^{i}-T_{c}^{i}\right)}{T_{0} \cdot\left(P_{\mathrm{HOT}}^{i}-P_{\mathrm{COLD}}^{i}\right)}+1-\frac{T_{c}^{i}}{T_{0}}-\frac{F_{2}\left(\Gamma_{\mathrm{OUT}}^{i}\right)}{G_{\mathrm{avTRT}}^{i}}$

where $\Gamma_{S}^{i}$ is the noise-source reflection coefficient referred to plane $1, T_{h}^{i}$ is the noise-source hot temperature (obtained from the noise-source calibrated ENR data) referred to plane $1, T_{c}^{i}\left(=T_{\mathrm{amb}}\right)$ is the noise-source cold temperature ambient temperature, $G_{\text {avTRT }}^{i}$ is the transistor available gain (computed from the transistor $S$-parameters and $\left.\Gamma_{S}^{i}\right)$, and $F_{2}\left(\Gamma_{\text {OUT }}^{i}\right)$ is the receiver noise figure for the FET output reflection coefficient $\Gamma_{\text {OUT }}^{i}$ (computed from the receiver noise parameters determined in the calibration). Introducing $F_{\mathrm{TRT}}\left(\Gamma_{S}^{i}\right)$ and $\Gamma_{S}^{i}$ in (5)-(10), together with the required $A B C D$, correlation and conversion matrices (computed from the FET equivalentcircuit extrinsic elements and $Y^{\mathrm{INT}}$ ), the system is solved for 


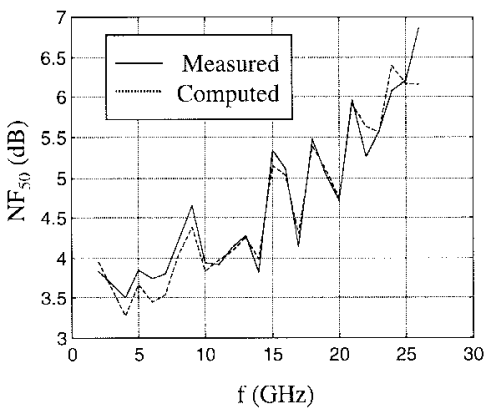

Fig. 5. Measured noise parameters of a $0.3-\mu \mathrm{m}$ gate length, $2 \times 50 \mu \mathrm{m}$ width HEMT. $\left(V_{\mathrm{ds}}=1.5 \mathrm{~V}\right.$. $\left.V_{\mathrm{gs}}=0.5 \mathrm{~V}\right)$. Comparison of $F_{50}$ measured $(-)$ and $F_{50}$ computed from the noise parameters measured with the new technique (- -).

the hybrid-noise matrix elements $C_{i j}^{\mathrm{INT}}$ at every bias point, as explained in Section II. The FET cascade noise matrix $C_{A}^{\mathrm{INT}}$ is computed using (3). Finally, the transistor noise parameters are obtained from well-known transformation formulas [8].

\section{VERIFICATION AND EXPERIMENTAL RESUlTS}

The noise-parameter measurement method proposed in this paper has been tested up to $26 \mathrm{GHz}$ on on-wafer high electronmobility transistors (HEMT's) with gate lengths of 0.3 and $0.5 \mu \mathrm{m}$, from the FhG-IAF DPD-SQW process (Foundry of Fraunhofer Institut, Freiburg, Germany). In this section, experimental results are presented, and a comparison of the novel method with tuner-based methods and other $F_{50}$ methods is given.

In Fig. 5, the accuracy with which the fitting algorithm adjusts the measured noise figure $F_{50}$ given by (11), to the noise figure $F_{50}$ computed using (1) from the measured noise parameters, is presented. The frequency range is $2-26 \mathrm{GHz}$. The FET is a $0.3-\mu \mathrm{m}$ device with a $2 \times 50 \mu \mathrm{m}$ gatewidth $\left(V_{\mathrm{gs}}=0.5 \mathrm{~V}, V_{\mathrm{ds}}=1.5 \mathrm{~V}\right)$. The agreement is excellent within the measurement accuracy (including noise meter and noise source) of about $\pm 0.15-0.2$-dB rms. This result supports the validity of the method proposed because the measurement of noise figure for a matched source $\left(F_{50}\right)$ is very insensitive to measurement uncertainties. Thus, the measured $F_{50}$ can be taken as a reference.

The transistor noise parameters determined from $F_{50}$ by solving (5)-(10), as explained in Section II, are compared to the noise parameters measured using the commercial NPTS from Cascade-Microtech ${ }^{1}$ based on a broad-band tuner. Fig. 6 plots the results as a function of frequency for the same FET and bias point as in Fig. 5. Fig. 7 shows two plots as a function of the drain bias-current $I_{d}$ at $12-$ and $18-\mathrm{GHz}$ frequencies, respectively. In both figures, the agreement is very good.

Fig. 8 plots the intrinsic hybrid noise matrix elements determined as a function of frequency for the same device as in Figs. 5-7. The element $C_{11}^{\mathrm{INT}}$ is a constant, as suggested in the literature [10], [12]-[14], while $\operatorname{Im}\left(C_{12}^{\mathrm{INT}}\right)$ is very small (also suggested in [17]), one order of magnitude smaller than $\operatorname{Re}\left(C_{12}^{\mathrm{INT}}\right)$, and almost constant. The best fitting of $F_{50}$ has been obtained when $\operatorname{Re}\left(C_{12}^{\mathrm{INT}}\right)$ is not neglected, in agreement with the literature [17], and a negative frequency
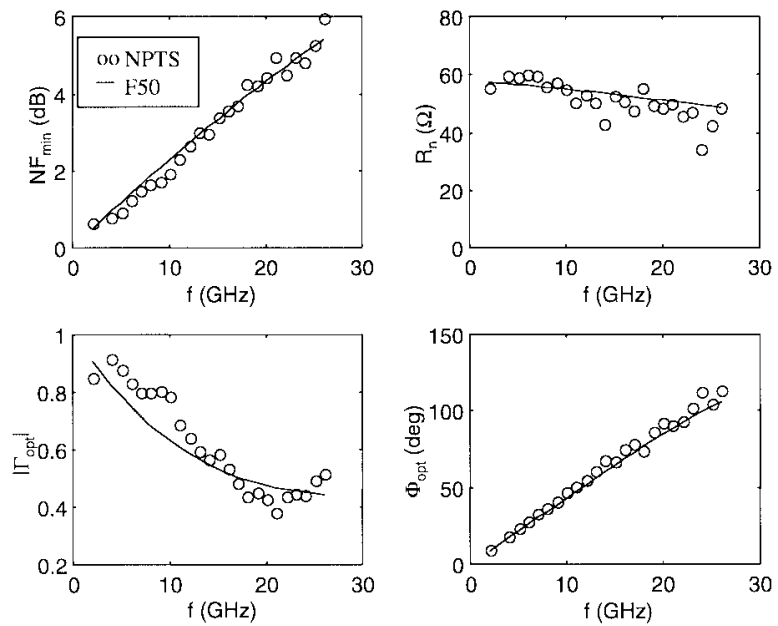

Fig. 6. Comparison of noise parameters directly measured using the commercial NPTS system ${ }^{1}$ (o) and noise parameters measured with the new technique $(-)$. The FET and bias point are the same as in Fig. 5.

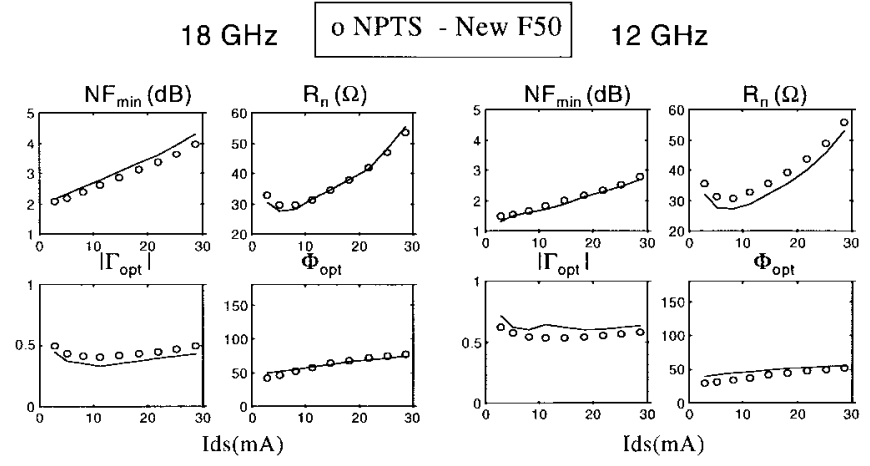

Fig. 7. Bias dependence of the noise parameters measured with the new $F_{50}$ method $(-)$ and NPTS $^{1}(\mathrm{o})$. The FET and bias point are the same as in Fig. $5\left(V_{\mathrm{ds}}=1.5 \mathrm{~V}\right)$.
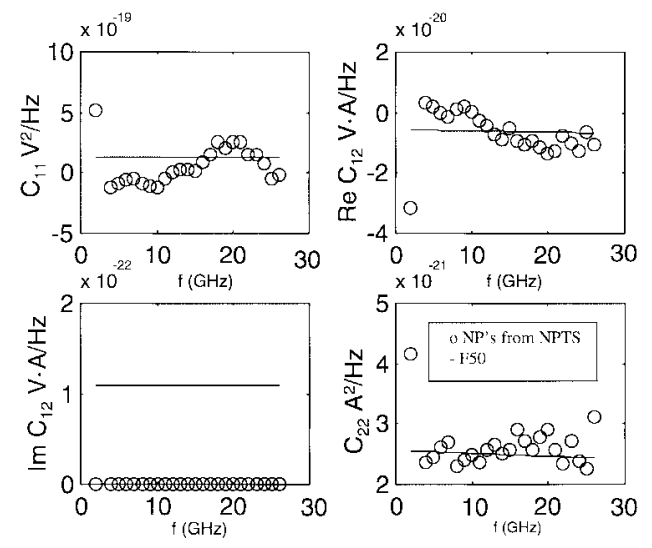

Fig. 8. Measured hybrid noise matrix elements with new $F_{50}$ method as a function of frequency $(-)$ and the extracted from noise parameters measured with the commercial NPTS system ${ }^{1}$ (o). The FET and bias point are the same as in Fig. 5.

slope is allowed for $C_{22}^{\mathrm{INT}}$ and $\operatorname{Re}\left(C_{12}\right)$. The frequency dependence obtained for $C_{22}^{\mathrm{INT}}$ is in accord to Monte Carlo device simulations [30]. This effect has also been observed 

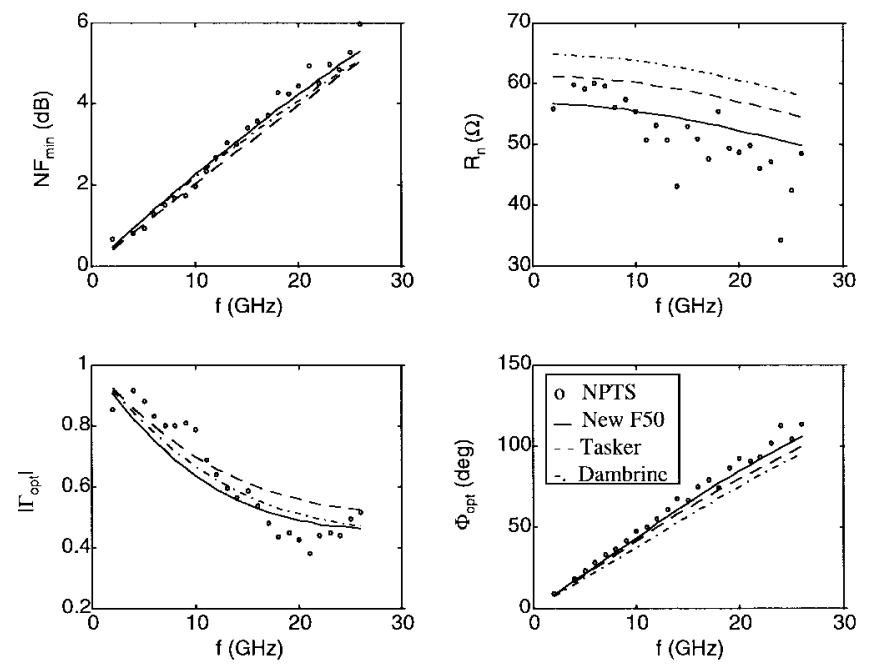

Fig. 9. Comparison of methods. Noise parameters of a 0.3- $\mu \mathrm{m}$ HEMT with $2 \times 50 \mu \mathrm{m}$ gatewidth measured with NPTS (o), new $F_{50}$ technique $(-), F_{50}$ method proposed by Dambrine [9] $(\cdot-\cdot), F_{50}$ method proposed by Tasker [12] $(--)$.

in the measurement of devices from other GaAs technologies (F20 MESFET from GEC-Marconi, D02AH pseudomorphic HEMT (pHEMT) from Philips, and other pHEMT's from CNRS).

Fig. 9 compares the results of the $F_{50}$ method proposed in this paper with those obtained by applying other $F_{50}$ methods previously published [9], [12] and the commercial system. ${ }^{1}$ The measured noise parameters of a $0.3-\mu \mathrm{m}$ device with a $2 \times 50 \mu \mathrm{m}$ gatewidth are shown. Main discrepancies between the three $F_{\tilde{z} 0}$ methods are observed in $\left|\Gamma_{\text {opt }}\right|$ and $R_{n}$. In the method proposed by Tasker et al. [12], the discrepancies can be explained because $C_{H}^{\mathrm{INT}}$ is neglected (Pospieszalski model [10]), and $C_{H}^{\mathrm{INT}}$ mainly affects $\left|\Gamma_{\text {opt }}\right|$ and $R_{n}$ [8], [14]. In the method proposed by Dambrine et al. [9], the correlation coefficient of the admittance model is assumed to be purely imaginary and related to parameters $R, P$ by the approximate expression $C \cong(R / P)^{1 / 2}$, and this assumption is equivalent to $C_{H}^{\mathrm{INT}}=0$ [10], [13].

An improvement in the determination of $T_{d}$ (and, consequently, the four noise parameters) when using the method proposed in [12] with the Pospieszalski model [10] is achieved by allowing a linear frequency dependence (linear regression) for $T_{d}$. This is equivalent to imposing the following constraints in (5): $C_{12}^{\mathrm{INT}}=0, C_{11}^{\mathrm{NT}}=4 k T_{a} R_{i} \Delta f, C_{22}^{\mathrm{INT}}=4 k T_{d} \Delta f / R_{\mathrm{ds}}$. Fig. 10 compares the results of estimating $T_{d}$ using the method proposed in [12], the method proposed in [12] plus a linear regression for $T_{d}$, the $F_{\tilde{\jmath} 0}$ method proposed in this paper, and the commercial method [4]. The frequency slope and values of $T_{d}$ obtained with linear regression are in a close agreement (within measurement uncertainties) with those obtained with the $F_{50}$ method proposed here.

The accuracy with which the transistor noise parameters are measured depends on a number of measurement variables, such as the ENR value and magnitude of $\Gamma_{\text {opt }}\left(\left|\Gamma_{\text {opt }}\right|\right)$, on uncertainties in the required measurements, such as $S$-parameters and noise powers, and the ENR calibrated-data uncertainty.

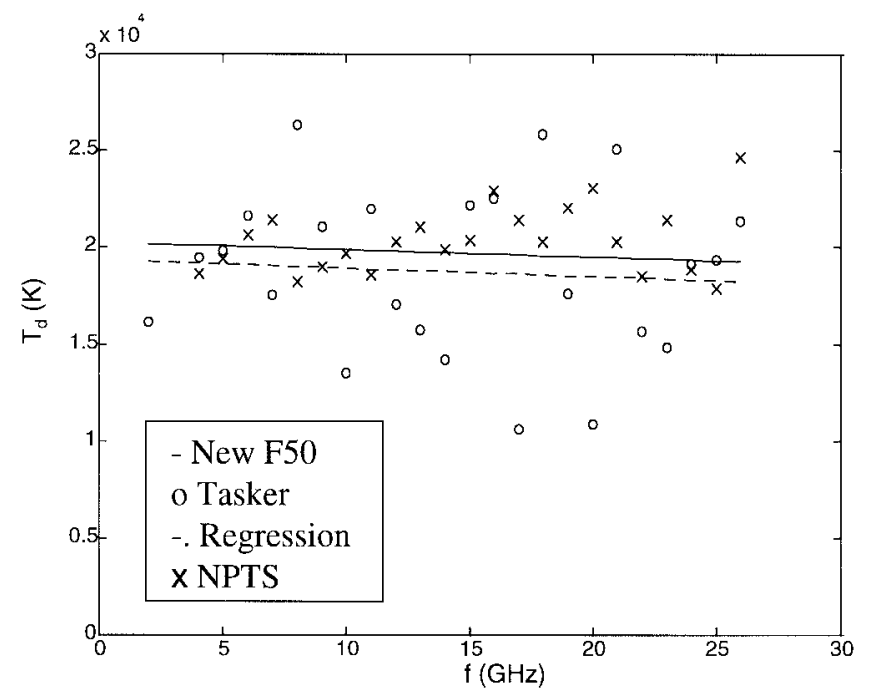

Fig. 10. Estimated drain temperature of a $0.3-\mu \mathrm{m}$ HEMT by using the new $F_{50}$ method $(-)$, the $F_{50}$ method proposed by Tasker [12] (o), (-), the $F_{50}$ method proposed by Tasker [12] plus a linear regression $(\cdot-\cdot)$, and the tuner-based commercial method $\operatorname{NPTS}^{1}(x)$.

Tuner-based methods are particularly sensitive to $\left|\Gamma_{\text {opt }}\right|$. In fact, they show a frequency ripple that becomes more significant at low frequencies (the same effect is observed at low bias drain-current) for which $\left|\Gamma_{\text {opt }}\right|$ is high. This effect can be observed in Figs. 6, 7, and 9. Consequently, the accuracy of tuner-based methods may decrease whenever $\left|\Gamma_{\text {opt }}\right|$ is high. In contrast, the $F_{50}$-based methods give a smooth noiseparameter frequency dependence because they are based on the fundamental assumption of a smooth intrinsic noise-source dependence of frequency.

To determine the sensitivity of tuner-based methods to $\left|\Gamma_{\text {opt }}\right|$, a Monte Carlo analysis was performed. The inputs to the Monte Carlo algorithm are real measurements (noise powers, $S$-parameters, and source reflection coefficients) obtained from the tuner-based system. The measurements are randomly perturbed within the measurement uncertainties. Uncertainties were estimated from the data provided by the ANA and NMS manufacturers, and by a direct measurement of reflectioncoefficient and noise power random fluctuations in a number of time series. The measured uncertainties in $|\Gamma|$ were $0.05 \%$ and $0.12 \%$, respectively for the ANA and NMS. Detector nonlinearity (evaluated from NMS manufacturer's data) and measured gain unstabilities were also taken into account in the simulator. The noise parameters were then determined from the perturbed data by a number of measurement methods [2], [3] and extraction procedures [5]-[7]. An important advantage of the Monte Carlo method is that no analytical expressions are required to compute the errors; specifically, errors are not combined as in rms or worst case (WC) classical analysis, and error propagation is intrinsically taken into account. Consequently, results of the error analysis are more. The results are presented in Fig. 11, in which the simulated error (rms) in the determination of the noise parameters is plotted as a function of $\left|\Gamma_{\text {opt }}\right|$ at $f=9 \mathrm{GHz}$. The errors show a quick increase as $\left|\Gamma_{\text {opt }}\right|$ is greater than the maximum source 

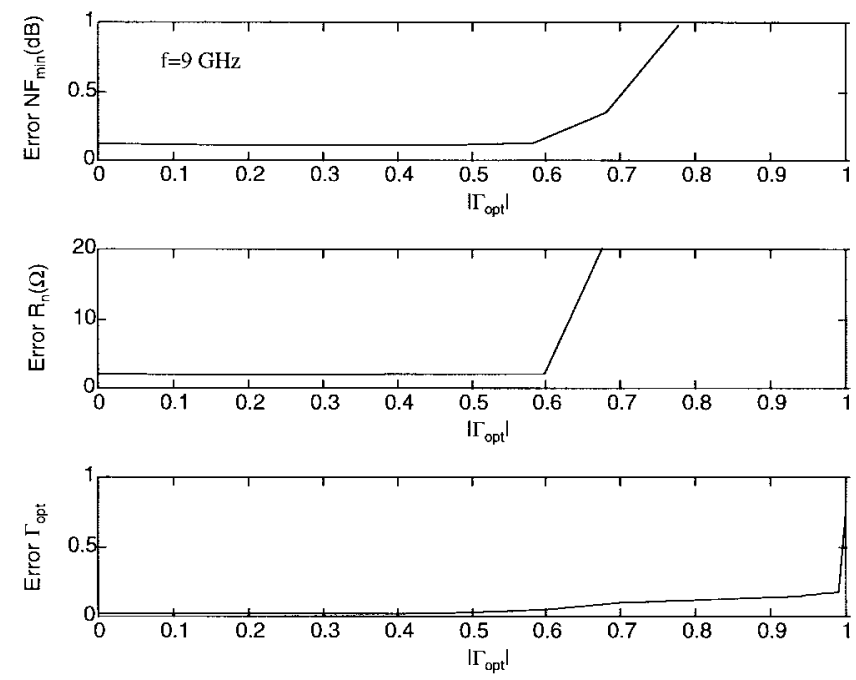

Fig. 11. The rms error in the noise-parameter measurement with tuner-based methods as a function of $\left|\Gamma_{\text {opt }}\right|$, computed from a Monte Carlo analysis, for a $0.3-\mu \mathrm{m}$ HEMT $(f=9 \mathrm{GHz})$.

reflection coefficient. This result is in agreement with other works [3]. The maximum source reflection-coefficient $(\sim 0.6$ in our case) is limited by the tuner itself and especially by the losses between the tuner plane $1^{\prime}$ and the input coplanar probe (plane 1). Error is particularly important in the lower frequency band $(f<10 \mathrm{GHz})$ for which $\left|\Gamma_{\text {opt }}\right|$ is very high (close to one). This effect can be recognized in the frequency dependence of the noise matrix extracted from the noise parameters measured with the tuner-based system (see Fig. 8). However, because the receiver exhibits a low $\left|\Gamma_{\text {opt }}\right|$ (about 0.15 in Fig. 4), tuner-based methods can be confidently used to determine the receiver noise parameters, as it was performed in Section III.

The measurement errors of $F_{50}$ methods are basically independent of $\left|\Gamma_{\text {opt }}\right|$ because the magnitude of the source coefficient $\left|\Gamma_{S}\right|$ is very low. They only depend on the gain fluctuations of the NMS setup, the ENR uncertainly, and the noise-detector nonlinearities. Taking into account all these effects, the simulated results show error values of about $\left(0.15-0.2-\mathrm{dB} \mathrm{rms}\right.$ in the measured $F_{50}$, up to $26 \mathrm{GHz}$, in agreement with the experimental results (Fig. 5). The standard deviation in the determination of the FET noise parameters has been computed from a Monte Carlo analysis (see Fig. 12). The values obtained are similar in all frequencies up to $26 \mathrm{GHz}$, and they are significantly lower than those obtained with tunerbased methods, in particular at low frequencies or for low-bias drain currents, for which $\left|\Gamma_{\text {opt }}\right|$ is high.

\section{Application to FET Noise Modeling}

Using the procedure described in Section II, the dependence of $C_{\text {egid }}^{\mathrm{INT}}$ on the frequency $(f)$ and the normalized drain-bias current $I_{d}\left(I_{d}=I_{\mathrm{ds}} / Z\right.$ where $I_{\mathrm{ds}}$ is the drain bias current and $Z$ is the FET gatewidth) can be easily derived. In this section, simple expressions for the intrinsic noise matrix elements $C_{11}^{\mathrm{INT}}, C_{22}^{\mathrm{INT}}$, and $C_{12}^{\mathrm{INT}}$ as a function of $f, I_{d}$, and $Z$ are proposed to produce a scalable noise model for the FET, which is useful in computer-aided design (CAD). These expressions
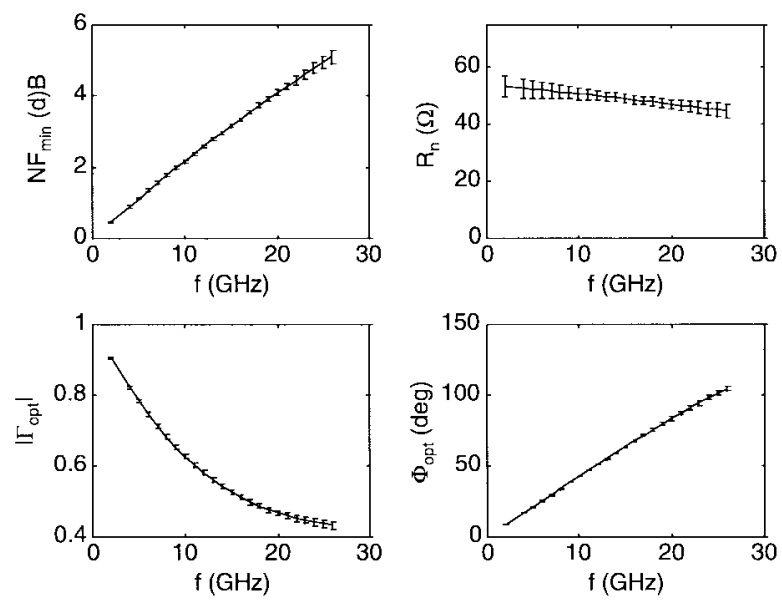

Fig. 12. Mean value and standard deviation of the noise parameters measured with new $F_{50}$ method from a Monte Carlo analysis.

are used in this section to obtain a particular model for the measured FET's.

Referring to Fig. 1, the rms value of the voltage noisesource $e_{g}\left(C_{11}^{\mathrm{INT}}\right)$ is proportional to $\left(R_{i} T_{g}\right)$ according to Pospieszalski's model [10]. $T_{g}$ is weakly dependent on $I_{d}$ and its value is close to room temperature [12], [15]. The following linear approximation can be used:

$$
T_{g}=T_{g 0}+T_{g 1} \cdot I_{d}
$$

The resistance $R_{i}$ is obtained from the measured $S$-parameters and the small-signal equivalent circuit. $C_{11}^{\mathrm{INT}}$ can then be expressed as

$$
C_{11}^{\mathrm{INT}}=4 k \Delta f \cdot \frac{R_{i}^{\prime}}{Z} \cdot T_{g}
$$

where $Z$ is the FET gatewidth and $R_{i}^{\prime}$ is the $R_{i}$ of a unit width. Alternatively, $C_{11}^{\mathrm{INT}}$ can be written in terms of the FET intrinsic cutoff frequency $\left(f_{T}=g_{m} /\left(2 \pi C_{\mathrm{gs}}\right)\right)$ [21]: $C_{11}^{\mathrm{INT}} \propto f_{T}^{-2}$.

According to the same model [10], the rms value of the current noise source $i_{d}\left(C_{22}^{\mathrm{INT}}\right)$ is proportional to $\left(T_{d} / R_{\mathrm{ds}}\right)$, where $T_{d}$ is nearly linear [11], and $R_{\mathrm{ds}}$ weakly dependent, on $I_{d}$. The same dependence of $C_{22}^{\mathrm{INT}}$ on $I_{d}$ is proposed in [21], where the following expression is given:

$$
\overline{i_{d}^{2}}=2 q \cdot \Delta f \cdot \eta^{2} \cdot I_{\mathrm{ds}}
$$

where $\eta$ is a technological parameter that depends on materials and the fabrication process. Alternatively, technologists may prefer to use the well-known Fukui semiempirical expression for $F_{\min }$ [22] in terms of a fitting factor $K_{f}$ that is related to $T_{d}$ by [11]

$$
K_{f}=\frac{2}{T_{o}} \sqrt{\frac{T_{d} T_{g}}{R_{\mathrm{ds}} g_{m}}}
$$

where $R_{\mathrm{ds}}$ and $g_{m}$ are obtained from the measured $S$ parameters and the small-signal equivalent circuit. In [23], another expression is proposed for $K_{f}$ in terms of $I_{d}$ and $Z$ 

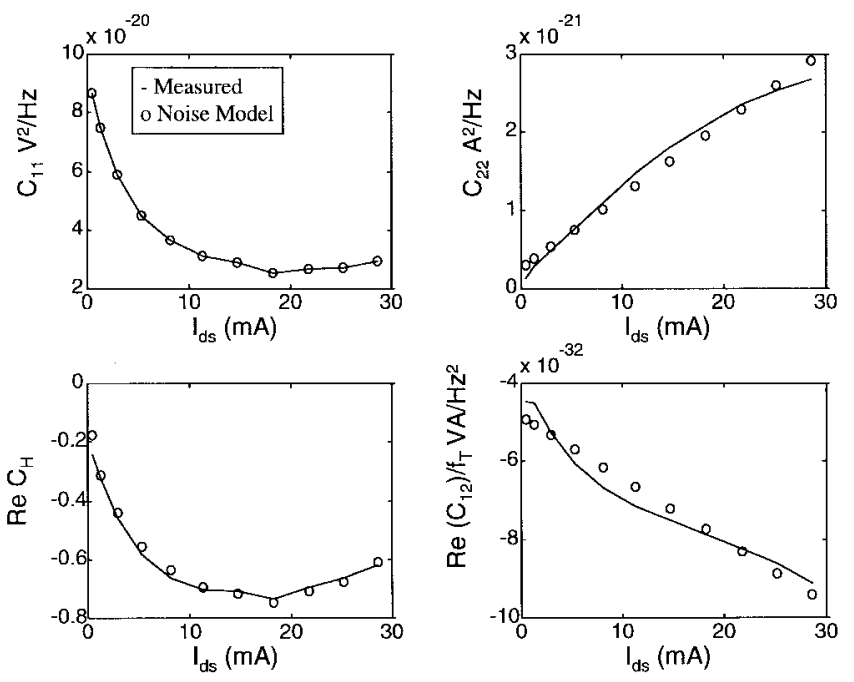

Fig. 13. Measured (-) and adjusted (from noise model) (o) drain current dependence of the hybrid noise matrix ( $f=20 \mathrm{GHz}$ ) for a $0.5-\mu$ m gate-length $2 \times 25 \mu \mathrm{m}$ gatewidth HEMT $\left(V_{\mathrm{ds}}=1.5 \mathrm{~V}\right)$.

as follows:

$$
K_{f}=2 \sqrt{\frac{L}{C_{\mathrm{gs}}}\left(\alpha \cdot Z+\beta \cdot I_{\mathrm{ds}}\right)}
$$

where $L$ is the gate length, and the technological parameters $\alpha, \beta$ are basically independent of the active-layer geometry.

Our experimental results (see Fig. 10 for comparison) show a very close agreement between $C_{22}^{\mathrm{INT}}$ obtained under no simplifying assumptions on the hybrid model $C_{\text {egid }}^{\mathrm{INT}}$, as proposed in this paper, and $C_{22}^{\mathrm{INT}}$ (or, equivalently, $T_{d}$ ) obtained using the expressions given by Pospieszalski [10] for the simplified hybrid model (in which the correlation coefficient is assumed zero). In other words, the arguments given in this section for the dependence of $C_{22}^{\mathrm{INT}}$ on $I_{d}$ are valid for the hybrid model (with no simplifying assumptions) proposed here. However, our results show that $C_{22}^{\mathrm{INT}}$ is also frequency dependent and that $C_{12}^{\mathrm{INT}}$ has an important effect in the determination of the FET noise parameters, thus, it should not be neglected. It is found that $C_{12}^{\mathrm{INT}}$ is basically real and that $\operatorname{Re}\left(C_{12}^{\mathrm{INT}}\right)$ is linearly dependent on $f_{T}$. Depending on the FET type, it is found that the best results in the determination of the FET noise parameters are obtained if a linear dependence on $f$ is allowed for $\operatorname{Re}\left(C_{12}^{\mathrm{INT}}\right)$ as well.

Consequently, the following frequency- and bias-currentdependent scalable model is proposed:

$$
\begin{aligned}
C_{11}^{\mathrm{INT}} & =4 k \Delta f \cdot \frac{R_{i}^{\prime}}{Z} \cdot\left(T_{g 0}+T_{g 1} \cdot I_{d}\right) \\
C_{22}^{\mathrm{INT}} & =4 k \Delta f \cdot Z \cdot\left[\left(a+b \cdot I_{d}\right)+\left(c+d \cdot I_{d}\right) \cdot f\right] \\
\operatorname{Re}\left(C_{12}^{\mathrm{INT}}\right) & =4 k \Delta f \cdot f_{T} \cdot\left(g+h \cdot I_{d}+\left(m+n \cdot I_{d}\right) \cdot f\right)
\end{aligned}
$$

where the unknown coefficients are fitted with a linear regression of $T_{g}, C_{22}^{\mathrm{INT}}$, and $\operatorname{Re}\left(C_{12}\right) / f_{T}$ versus the normalized drain current $I_{d}$.

Although this model has been obtained from the measurement of a number of particular HEMT devices, as explained
TABLE II

Noise Model for 0.5-mm Gate-Length HEMT Devices

$$
\begin{aligned}
& \left(I_{d}=I_{\mathrm{ds}} / Z \mathrm{IN} \mathrm{A} / \mathrm{m}, f \mathrm{IN} \mathrm{GHz}, Z \mathrm{IN} m, R_{i}^{\prime}=R_{i} \cdot Z \mathrm{IN} \Omega \cdot m\right) \\
& \text { CAD Model for } 0.5 \mu \mathrm{m} \text { gate-length FhG IAF HEMT } \\
& C_{11}^{\text {NTT }}\left(V^{2} / H z\right)=4 k \cdot \frac{R_{i}^{\prime}}{Z} \cdot\left(0.244 \cdot I_{d}+400.89\right) \\
& C_{12}^{I N T}(V \cdot A / H z)=4 k \cdot f_{T} \cdot\left(0.0035 \cdot I_{d}-0.6483\right) \times 10^{-8} \\
& C_{22}^{I N T}\left(A^{2} / H z\right)=4 k \cdot Z \cdot\left(\left(0.1212 \cdot I_{d}+2.3058\right) \times 10^{4}+\left(-0.0029 \cdot I_{d}-0.8502\right) \times 10^{-6} \cdot f\right)
\end{aligned}
$$
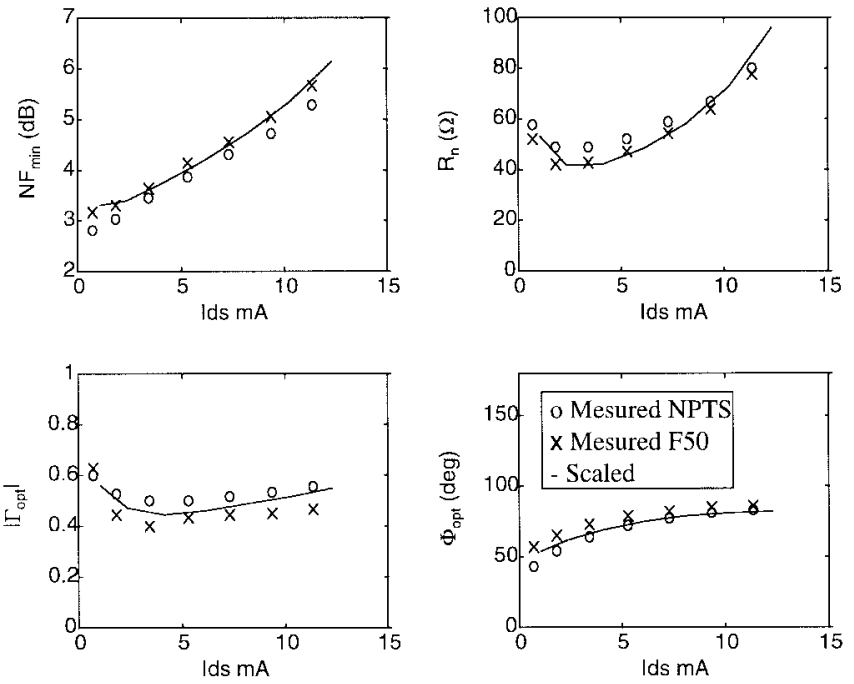

Fig. 14. Noise parameters of a $0.5-\mu \mathrm{m}$ device with $2 \times 50 \mu \mathrm{m}$ gatewidth measured (o) and computed from the noise model $(-)$ obtained from a $2 \times 25 \mu \mathrm{m}$ gatewidth device $(f=26 \mathrm{GHz})$.

in Section V, it can be used for any FET in the microwave range because it is based on physical considerations of the dependence of the noise sources on $I_{d}$ and frequency. Alternatively, it can be formulated for other intrinsic noise-source configurations, for example the admittance (current-current) configuration [9], by allowing an appropriate frequency and drain current dependence of the intrinsic noise sources based on physical considerations [16].

Fig. 13 shows the drain bias-current dependence of the measured hybrid noise matrix and the adjusted model using (16)-(19). The drain noise-current density $C_{22}^{\mathrm{INT}}$ is a linear function and the voltage density $C_{11}^{\mathrm{INT}}$ depends on the intrinsic cutoff frequency $f_{T}$ as $\left(1 / f_{T}^{2}\right)$. Therefore, it decreases with $I_{d} \cdot \operatorname{Re}\left(C_{12}^{\mathrm{INT}}\right) / f_{T}$ is a linear function of $I_{d}$. It is clearly seen that the correlation coefficient $C_{H}^{\mathrm{INT}}$ cannot be neglected, in particular, for high drain bias currents. To the authors' knowledge, the results obtained for $C_{H}^{\mathrm{INT}}$ have not been published before.

To verify the scaling capability of the proposed model, the measured intrinsic noise-matrix of a $0.5-\mu \mathrm{m}$ device with a $2 \times 25 \mu \mathrm{m}$ gate length was used to compute the noise parameters of a $0.5-\mu \mathrm{m}$ device with a different gate length (2 $\times 50 \mu \mathrm{m})$. Table II shows the model for the $0.5-\mu \mathrm{m}$ devices obtained by fitting the coefficients in (16)-(19) to the measured intrinsic matrix. Fig. 14 compares the scaled noise parameters 
of the $2 \times 50 \mu \mathrm{m}$ device to those directly measured by using two different methods, $F_{50}$ proposed here, and the commercial tuner-based system. ${ }^{1}$ The noise parameters are plotted as a function of $I_{d}$ at the 26-GHz frequency, and show a good agreement which supports the self-consistency of the model.

\section{CONCLUSION}

A mathematical method for determining the noise matrix $C_{\text {egid }}^{\text {INT }}$ of FET's based on the measurement of $F_{50}$ for a redundant number of frequency points has been proposed and successfully applied to HEMT's. By assuming a linear frequency dependence (higher order polynomials have also been considered with no significant improvement of results) for the elements of $C_{\text {egid }}^{\mathrm{INT}}$, an excellent agreement between the computed $F_{50}$ and the measured $F_{50}$ up to $26 \mathrm{GHz}$ is achieved. The noise matrix elements obtained, and their frequency dependence, agree with results previously published by other authors. An enhanced accuracy (compared to other $F_{50}$ methods in the literature) in the determination of the FET noise parameters is obtained if $\operatorname{Re}\left(C_{12}^{\mathrm{INT}}\right)$ is not neglected and a linear frequency dependence is included in $\operatorname{Re}\left(C_{12}^{\mathrm{INT}}\right)$ and $C_{22}^{\mathrm{INT}}$. An automatic tuner is not required to perform the receiver full-noise calibration, but only a versatile "manual tuner," commonly found in a microwave laboratory, which produces the same accurate results. Theoretical Monte Carlo analysis and experimental results show an enhanced accuracy of the new method compared to tuner-based methods, in particular, whenever $\left|\Gamma_{\text {opt }}\right|$ is high (low frequencies or lowbias drain currents) because the new method is basically independent of $\left|\Gamma_{\text {opt }}\right|$. To summarize, the new $F_{50}$ method proposed here is a fast and simple technique that overcomes the limitations of conventional tuner-based methods for the determination of the noise parameters of an FET, providing both an enhanced accuracy and useful device noise models.

\section{REFERENCES}

[1] R. Q. Lane, "The determination of device noise parameters," Proc. IEEE, vol. 57, pp. 1461-1462, 1969.

[2] V. Adamian and A. Uhlir, "A novel procedure for receiver noise characterization," IEEE Trans. Instrum. Meas., vol. IM-22, pp. 181-182, Feb. 1973.

[3] G. Caruso and M. Sannino, "Computer-aided determination of microwave two-port noise parameters," IEEE Trans. Microwave Theory and Tech., vol. MTT-26, pp. 639-642, Sept. 1978.

[4] M. Sannino, "On the determination of device noise and gain parameters," Proc. IEEE, vol. 67, pp. 1364-1366, Sept. 1979.

[5] A. C. Davidson, B. W. Leake, and E. Strid, "Accuracy improvements in microwave noise-parameter measurements," IEEE Trans. Microwave Theory Tech, vol. 37, pp. 1973-1978, Dec. 1989.

[6] J. M. O'Callaghan and J. P. Mondal, "A vector approach for noise parameter fitting and selection of source admittances," IEEE Trans. Microwave Theory Tech., vol. 39, pp. 1376-1382, Aug. 1989.

[7] J. M. O'Callaghan, A. Alegret, L. Pradell, and I. Corbella, "Ill conditioning loci in noise parameter determination," Electron. Lett., vol. 32, no. 18, pp. 1680-1681, 1996.

[8] H. Hillbrand and P. H. Russer, "An efficient method for computer aided noise analysis of linear amplifier networks," IEEE Trans. Circuits Syst., vol. CAS-23, pp. 235-238, Apr. 1976.

[9] G. Dambrine, H. Happy, F. Danneville, and A. Cappy, "A new method for on wafer noise measurement," IEEE Trans. Microwave Theory Tech., vol. 41, pp. 375-381, Mar. 1993.

[10] M. Pospieszalski, "Modeling of noise parameters of MESFET's and MODFET's and their frequency and temperature dependence," IEEE Trans. Microwave Theory Tech., vol. 37, pp. 1340-1350, Sept. 1989.
[11] __ "FET noise model and on-wafer measurement of noise parameters," in IEEE MTT-S Dig., 1991, pp. 1117-1120.

[12] P. J. Tasker, W. Reinert, B. Hughes, J. Braunstein, and M. Schlechtweg, "Direct extraction of all four transistor noise parameters using a $50-\Omega$ measurement system," in IEEE MTT-S Int. Microwave Symp., 1993.

[13] F. Danneville, H. Happy, G. Dambrine, J. Belquin, and A. Cappy, "Microscopic noise modeling and macroscopic noise models: How good a connection?," IEEE Trans. Electron Devices, vol. 41, pp. 779-785, May 1994.

[14] R. A. Pucel, "A general noise de-embedding procedure for packaged two-port linear active devices," IEEE Trans. Microwave Theory Tech., vol. 40, pp. 2013-2020, Nov. 1992

[15] M. S. Gupta and P. T. Greiling, "Microwave noise characterization of GaAs MESFET's, determination of extrinsic noise parameters," IEEE Trans. Microwave Theory Tech., vol. 36, pp. 745-751, Apr. 1988.

[16] A. Gasmi, B. Huyart, E. Bergeault, and L. P. Jallet, "A new calculation approach of transistor noise parameters as a function of gatewidth and bias current," IEEE Trans. Microwave Theory Tech, vol. 45, pp. 338-344, Mar. 1997.

[17] H. Jong-Hee and K. Lee, "A new extraction method for noise sources and correlation coefficient in MESFET," IEEE Trans. Microwave Theory Tech, vol. 44, pp. 487-492, Mar. 1996.

[18] A. Lázaro, L. Pradell, A. Beltrán, and J. M. O’Callaghan, "Direct extraction of all four transistor noise parameters from $50-\Omega$ noise figure measurements," Electron. Lett., vol. 34, no. 3, pp. 289-291, 1998.

[19] H. Kondoh, "An accurate FET modeling from measured $S$-parameters," in IEEE MTT-S Int. Microwave Symp. Dig., Baltimore, MD, 1986, pp. $377-380$.

[20] W. H. Press, B. P. Flannery, S. A. Teukolsky, and W. T. Vetterling, Numerical Recipes. Cambridge, U.K.: Cambridge Univ. Press, 1987.

[21] R. Reuter, S. Van Waasen, D. Peters, U. Auer, W. Brockerhoff, and F. J. Teguda, "A new temperature noise model of HFET with special emphasis on gate-leakage current and investigation of the bias dependence of the equivalent noise sources," in European Microwave Conf., 1995, pp. $205-210$.

[22] H. Fukui, "Design of microwave GaAs MESFET's for broad-band low noise amplifiers," IEEE Trans. Microwave Theory Tech., vol. MTT-27, pp. 643-650, 1979

[23] A. Cappy, "Noise modeling and measurement techniques," IEEE Trans. Microwave Theory Tech, vol. MTT-36, pp. 1-10, Jan. 1985.

[24] A. Davidson, K. Jones, and E. Strid, "LRM and LRRM calibration with automatic determination of load inductance," in 36th ARFTG Conf. Dig., Nov. 1990, pp. 57-62.

[25] P. Crozat, C. Boutex, M. Chaubet, V. Danelon, A. Sylvestre, and G. Vernet, " $50-\Omega$ noise measurements with full receiver calibration without tuner," Electron. Lett., vol. 32, no. 3, pp. 261-262, 1996.

[26] M. Berroth and R. Bosch. "High-frequency equivalent circuit of GaAs FET's for large-signal applications," IEEE Trans. Microwaves Theory Tech., vol. 39, pp. 224-229, Feb. 1992

[27] A. N. Riddle and R. J. Trew, "Low frequency noise measurements of GaAs FET's," in IEEE MTT-S Int. Microwave Symp. Dig., Baltimore, MD, 1986, pp. 79-82.

[28] A. Cappy, "Device physics and noise models," in IEEE MTT-S Int Microwave Symp. Workshop WFF, San Francisco CA, 1996.

[29] A. Lázaro, L. Pradell, and J. M. O’Callaghan, "Method for measuring noise parameters of microwave two-ports," Electron. Lett., vol. 34, no. 13, pp. 1332-1333, 1998.

[30] T. González, D. Pardo, L. Varani, and L. Reggiani, "Monte Carlo analysis of the behavior and spatial origin of electronic noise in GaAs MESFET's," IEEE Trans. Electron Devices, vol. 42, pp. 991-998, May 1995.

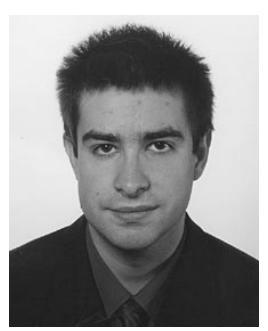

Antonio Lázaro was born in Lleida, Spain, in 1971 $\mathrm{He}$ received the M.Sc. degree in telecommunication engineering from the Polytechnic University of Catalonia (UPC), Barcelona, Spain, in 1994, and is currently working toward the Ph.D. degree at the same university.

His research interests are in HEMT device modeling, on-wafer noise measurement techniques, and monolithic microwave integrated circuits (MMIC's). 


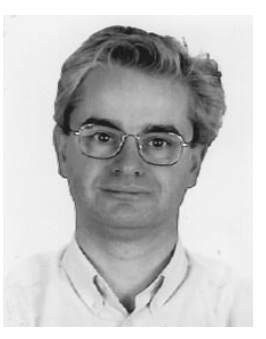

Lluís Pradell (S'87-M'89) was born in Barcelona, Spain, in 1956. He received the telecommunication engineering and Dr. degrees from the Polytechnic University of Catalonia (UPC), Barcelona, Spain, in 1981 and 1989 , respectively.

From 1981 to 1985, he was with Mier Allende, Barcelona, Spain, as an RF \& Microwave System Design Engineer. In 1985, he joined the faculty at UPC. Since 1985, he has been teaching courses on microwave circuits and antennas, and performing research on microwave devices and systems, onwafer network analyzer calibration techniques, noise-parameter determination, and modeling of MESFET's and HEMT's.

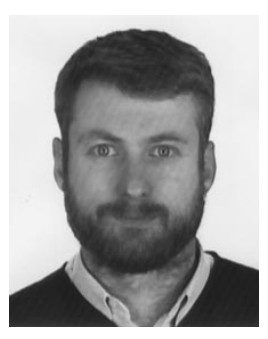

Juan M. O'Callaghan was born in Tortosa, Spain, in 1963 . He received the telecommunication engineering degree from the Polytechnic University of Catalonia (UPC), Barcelona, Spain, in 1987, and the M.S. and Ph.D. degrees from the University of Wisonsin at Madison.

From July to December 1989, he was an Intern at Honeywell's Systems Research Center. In 1993, he jointed the faculty at UPC, where he has been teaching courses on radar and high-frequency devices and systems and performing research on microwave devices and systems, high-frequency transistor characterization, and superconducting devices. 